\title{
Marc OUIMET
}

CRIMINOLOGUE, ÉCOLE DE CRIMINOLOGIE, UNIVERSITÉ DE MONTRÉAL

\author{
(1994)
}

\section{"La violence n'augmente plus! Quelques propositions pour en diminuer l'incidence”}

\author{
Un document produit en version numérique par Jean-Marie Tremblay, bénévole, \\ professeur de sociologie au Cégep de Chicoutimi \\ Courriel: jean-marie tremblay@uqac.ca \\ Site web pédagogique : http://www.uqac.ca/jmt-sociologue/
}

Dans le cadre de la collection: "Les classiques des sciences sociales"

Site web: http://classiques.uqac.ca/

Une collection développée en collaboration avec la Bibliothèque Paul-Émile-Boulet de l'Université du Québec à Chicoutimi Site web: http://bibliotheque.uqac.ca/ 
Cette édition électronique a été réalisée par Jean-Marie Tremblay, bénévole, professeur de sociologie au Cégep de Chicoutimi à partir de :

Marc OUIMET

\section{"La violence diminue! Quelques propositions pour ac- centuer cette tendance".}

Un article publié dans la revue Policy Options . v 15, n 8, 1994.

M Marc Ouimet, criminologue, nous a accordé le 11 septembre 2006 son autorisation de diffuser électroniquement cet article.

Courriel : marc.ouimet@umontreal.ca ou ouimetm@ere.umontreal.ca

Polices de caractères utilisée :

Pour le texte: Times New Roman, 14 points.

Pour les citations : Times New Roman, 12 points.

Pour les notes de bas de page : Times New Roman, 12 points.

Édition électronique réalisée avec le traitement de textes Microsoft Word 2004 pour Macintosh.

Mise en page sur papier format

LETTRE (US letter), 8.5”’ x 11'’)

Édition numérique réalisée le 21 novembre 2006 à Chicoutimi, Ville de Saguenay, province de Québec, Canada.

\section{f Fait avec}




\section{Marc Ouimet (1994)}

\section{"La violence diminue! Quelques propositions pour accentuer cette tendance”}

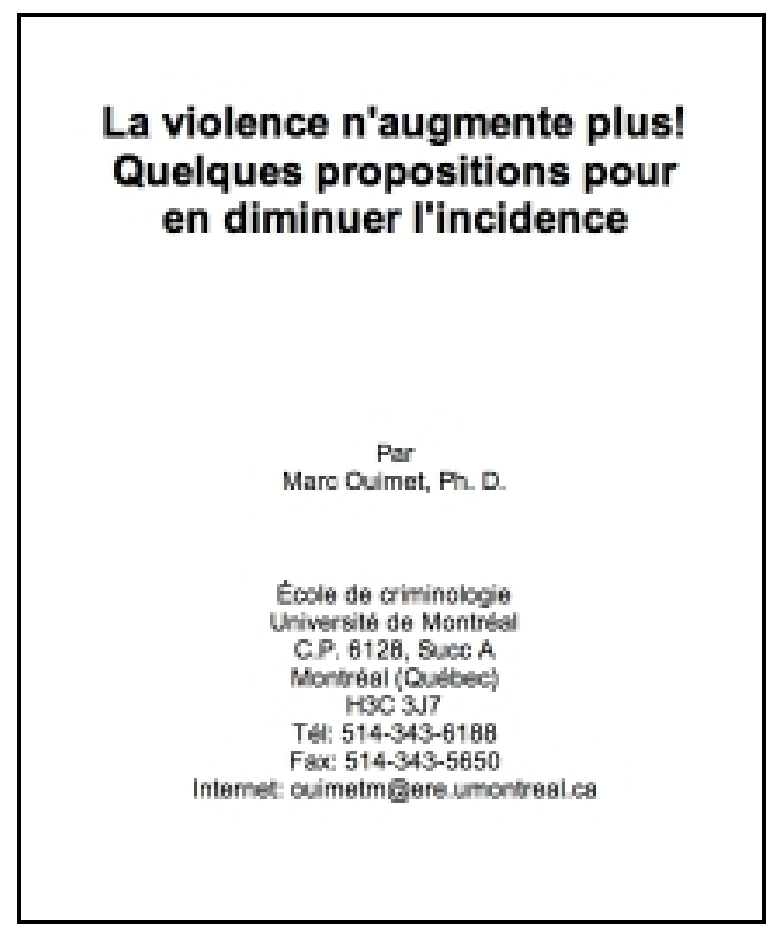

Un article publié dans la revue Policy Options . v 15, n 8, 1994. 


\section{Table des matières}

\section{Introduction}

Les tendances de la criminalité au cours des 30 dernières années

Quelques propositions pour limiter l'incidence de la criminalité 
Marc OUIMET 1

\section{"La violence diminue! Quelques propositions pour accentuer cette tendance".}

Un article publié dans la revue Policy Options . v 15, n 8, 1994.

\section{Introduction}

$\underline{\text { Retour à la table des matières }}$

Le crime façonne la vie de bien des gens. Certains, exaspérés par une réalité criminelle qu'ils jugent de plus en plus intense dans leur arrondissement, décident de s'établir dans une banlieue paisible. D'autres se procurent à fort prix un système d'alarme pour protéger leur résidence, ce qui du coup réduit sensiblement leurs primes d'assurances. Mais tous lisent attentivement les détails de la dernière affaire criminelle apparaissant à la une de leur quotidien. Puisque la réalité criminelle a un impact sur l'ensemble de la vie sociale, il est normal que les politiciens et décideurs cherchent à proposer des solutions visant à mieux contrôler la criminalité. Selon nous, les changements de nature administratifs sont plus efficaces que les changements législatifs lorsqu'il s'agit d'attaquer la criminalité.

1 Marc Ouimet est professeur agrégé de criminologie à l'université de Montréal et chercheur associé au Centre International de Criminologie de la même université. Cet article est basé sur une recherche financée par le FCAR et le CRSH portant sur les tendances et configurations de la criminalité. 
Dans le brouhaha quotidien entourant la question criminelle, il est difficile de bien voir quelles sont les tendances de fond qui la caractérise. Nous allons ici brosser un rapide tableau de l'évolution de la criminalité au Canada au cours des trente dernières années. Ensuite, nous apporterons des éléments d'explication sur les tendances observées. Finalement, nous proposerons un ensemble d'orientations qui permettraient de limiter le problème criminel dans notre société.

\section{Les tendances de la criminalité au cours des 30 dernières années}

$\underline{\text { Retour à la table des matières }}$

Des données fiables sur la criminalité au Canada sont disponibles depuis 1962, année où la collecte de l'information fut standardisée pour l'ensemble du pays. Les statistiques criminelles, ou policières, furent maintes fois critiquées: certains disent qu'elles mesurent plus l'activité de la police et des tribunaux que le crime lui-même. Puisque les biais caractérisant le ratio entre le nombre de crimes réellement commis et ceux connus sont relativement systématiques, les statistiques criminelles peuvent donc faire l'objet de comparaisons dans l'espace et dans le temps. De plus, les mesures alternatives de la criminalité sont loin d'être parfaites. Par exemple, les sondages nationaux de victimisation sont peu nombreux au Canada (il y en a eu un en 1982 pour les villes et un sondage national en 1987 et 1992) et comptent des échantillons à notre avis trop petits pour permettre une interprétation raisonnable des tendances. ${ }^{2}$

La figure 1 illustre les tendances de l'homicide, des voies de fait et de l'introduction avec effraction au Canada entre 1962 et 1992. Ces trois délits illustrent bien la diversité des types de crimes qui préocupent le public. De plus, les données sur l'homicide représentent la

2 Contrairement aux sondages en matière d'opinion politique, peu de gens sont annuellement exposés à un crime sérieux. Ainsi, pour obtenir un échantillon représentatif d'un événement rare, il faut compter sur un échantillon énorme. 
presque totalité des crimes de ce type commis, ce qui en fait un indicateur privilégié du niveau de violence présent dans notre société.

\section{INSERER LA FIGURE 1 ICI (non disponible)}

Les tendances indiquées à la figure 1 montrent que les années soixante et soixante-dix furent des années d'explosion de la criminalité. On peut dire que la plupart des activités criminelles sont devenues deux ou trois fois plus fréquentes en 1980 qu'en 1962. Cette hausse spectaculaire et incontestable de la criminalité est due à plusieurs facteurs ${ }^{3}$ :

- augmentation relative du nombre de jeunes dans la population,

- érosion du contrôle social informel (i.e. le blâme, la désaprobation morale...),

- érosion du contrôle social formel (i.e. diminution des probabilités d'arrestation et de condamnation),

- croissance économique soutenue (les opportunités criminelles devinrent de plus en plus nombreuses, alors que les biens devinrent de moins en moins bien gardés),

- une baisse de la gravité réelle de certains types de victimisations (les vols sont maintenant compensés par les assurances, les soins médicaux sont devenus gratuits et de bonne qualité).

Depuis 1980, la plupart des activités criminelles ont plafonné ${ }^{4}$. Ainsi, les hausses et baisses de la criminalité régulièrement annoncées par les agences policières et gouvernementales depuis cinq ans témoignent plus d'un plafonnement de la criminalité que de mouvements significatifs dans une direction ou l'autre. On pourrait même lancer l'hypothèse selon laquelle la criminalité a commencé à régresser au

3 Voir entre autres, Cusson Maurice, 1990, Croissance et décroissance du crime. Paris: Presses Universitaires de France.

4 Pour une analyse de l'ensemble de la question, voir Ouimet Marc, 1994, Les tendances de la criminalité apparente et de la réaction judiciaire au Québec: 1962 à 1991. In: Szabo Denis et Marc LeBlanc, Traité de criminologie empirique. Montréal: Les Presses de l'Université de Montréal. 
Canada. ${ }^{5}$ Mais à quoi pourrait être due ce plafonnement ou cette baisse? Ici, nous pourrions reprendre à l'envers la liste des facteurs ayant été exposés pour expliquer les hausses des décennies précédentes. Par exemple, la diminution de l'importance relative des 15-25 ans (qui sont passés de près de $20 \%$ de la population en 1980 à environ $14 \%$ maintenant) a certainement contribué à la tendance récente. D'ailleurs, on remarque qu'il y a un vieillissement de l'âge moyen de la population carcérale au Canada. Il y a donc eu un effet de cohorte important: la cohorte la plus criminalisée fut celle des adolescents du milieu des années soixante-dix. Nous pourrions aussi penser qu'il y a eu une amélioration de contrôle social informel au cours de la dernière décennie: on tolère de moins en moins les gestes de violence.

Si dans l'ensemble le portrait est clair, certains délits ne se comportent pas comme les autres. Par exemple, la courbe des voies de fait de la figure 1 montre que ce type de crime (idem pour les agressions sexuelles) serait devenu plus fréquent au cours des quinze dernières années. Nos travaux sur la signification réelle de cette observation montrent que deux processus jouent simultanément, soit qu'il y a une plus grande reportabilité (i.e. que les victimes rapportent plus souvent à la police ce qui leur est arrivé), et qu'il y a une plus grande prévalence du phénomène ${ }^{6}$. Mais pourquoi les agressions sexuelles et les voies de fait seraient-elles plus fréquentes alors que les autres formes d'activité criminelles auraient plafonnées ? Nous pensons que c'est encore l'effet démographique qui joue. En effet, si la propension au vol (introduction avec effraction, vol de véhicule à moteur...) est la plus forte entre 15 et 25 ans, la propension à l'expression pure de la violence est elle plus forte durant la trentaine. Ainsi, l'arrivée dans la trentaine de la cohorte hautement criminalisée des baby-boomers (particulièrement ceux nés entre 1955 et 1960) a eu un impact à la hausse sur ce type ce crime.

5 Notons qu'au moment de la rédaction de cet article, le Ministère de la sécurité publique du Québec annonçait que les statistiques de l'année 1993 montraient des baisses significatives pour plusieurs catégories d'activités criminelles sérieuses.

6 Voir: Ouimet Marc et Pierre Tremblay, 1994, Commentaire sur l'état de la criminalité au Québec. In: Statistique 1992: Criminalité et application des règlements de la circulation. Ministère de la sécurité Publique, Gouvernement du Québec. 
De plus, il semble que la délinquance juvénile ne suive pas les patterns observés pour l'ensemble de la société. En effet, une étude menée au Québec montre que le taux de délinquance juvénile (i.e. le nombre de jeunes mis en accusation par tranche de 1,000 jeunes) ait augmenté récemment pour un certain nombre de délits, malgré le fait que l'importance relative dans le contingent pénal diminue ${ }^{7}$. Une augmentation de la violence chez les jeunes serait donc réelle et doit être prise au sérieux. L'étude des causes de la délinquance juvénile montre que le facteur criminogène le plus puissant reste celui de la famille ${ }^{8}$. C'est la famille qui, par une socialisation adéquate du jeune, prévient en premier lieu le processus de criminalisation qui pourrait prendre place. La famille est d'abord responsable du développement de la personnalité du jeune et est ensuite celle qui appuiera le jeune dans son projet scolaire. Or, les familles brisées ou monoparentales montrent plus de difficulté dans les tâches requises pour une socialisation effective du jeune. En outre, la supervision des jeunes -- et en particulier des garçons -- devient de plus en plus difficile lorsque la mère est seule et souvent pauvre (la monoparentalité pousse souvent la mère vers l'assistance sociale).

Puisque les séparations en tous genres et la croissance du nombre de familles initialement monoparentales devinrent de plus en plus nombreux vers la fin des années soixante dix et le début des années quatre-vingt, les adolescents d'aujourd'hui proviennent disproportionnellement de familles désunies. Ainsi, l'absence du père se fait sentir par une plus grande difficulté des jeunes garçons d'aujourd'hui à s'adapter aux exigences de la vie contemporaine. Cette structure familiale, la monoparentalité, ne se répartit pas aléatoirement sur le territoire. Selon les données du recensement de 1991, dans certains secteurs de recensement de Montréal, jusqu'à 69\% des familles avec un enfant entre de moins de 18 ans à la maison seraient monoparentales !

7 Idem note infrapaginale 6.

8 Voir, par exemple, Gottfredson Michael et Travis Hirschi, 1990, A General Theory of Crime. Stanford, Ca: Stanford University Press. 


\section{Quelques propositions pour limiter l'incidence de la criminalité}

$\underline{\text { Retour à la table des matières }}$

Si la criminalité a plafonné ou diminue au Canada, il n'y a pas lieu de laisser les choses aller. Nous croyons plutôt que c'est à ce moment qu'il faut agir de manière à faire pointer les courbes de la criminalité définitivement vers le bas. Nous rappelons que si la criminalité au Canada est marginalement moins élevée que celle observée aux ÉtatsUnis, elle est encore bien plus fréquente que celle de la plupart des pays européens comparables (i.e. France, Suisse, Hollande...).

Pour plusieurs, on ne pourra faire baisser le niveau de violence et de vol de notre société qu'en s'en prenant aux causes profondes de la délinquance, soit la pauvreté, les toxicomanies, etc... Toutefois, comme l'a montré Charles Murray dans Losing Ground ${ }^{9}$, il n'y a pas de relation directe entre les montants alloués à la lutte contre la pauvreté et les effets de la pauvreté. A notre avis, la lutte à la criminalité doit être fondée sur un ensemble de petits combats bien ciblés, qui s'attaquent tant à l'organisation de notre réponse face au crime qu'aux causes prochaines des crimes.

Tout d'abord, c'est la famille qui devrait faire l'objet de stratégies de changement. Il faut réhabiliter la famille comme noyau central de la société. Les politiques sociales (en matière d'aide sociale) et économiques (en matière de taxation sur le revenu) devraient tendre à renforcer l'unité familiale. De plus, des programmes de développement d'habilité parentales des familles à risque pourraient être étendus. Mais le meilleur moyen d'influencer sur le désir de famille reste celui provenant de la sphère morale. Il reste toutefois difficile d'influencer les valeurs des citoyens sans entrer dans une croisade moraliste.

9 Charles Murray, 1984, Losing Ground: American social policy: 1950-1980. New York: Basic Books. 
D'autre part, la criminalité diminuera si les coûts associés aux conduites criminelles augmentent. Il s'agit ici de miser sur une plus grande efficacité de la police à identifier et accuser les personnes ayant commis des gestes de vol ou de violence. Nous pensons que les corps de police auraient avantage à rediriger une partie de leurs ressources sur le développement technologique. Par exemple, un fichier central d'agresseurs d'enfants permettrait d'augmenter les risques que courent ces délinquants d'être identifiés en cas de récidive, ce qui en ferait réfléchir plus d'un avant d'agir. De manière plus générale, la technologie (systèmes d'alarme, monnaie de plastique...) créera une pression à la baisse sur le nombre d'opportunités criminelles. A titre illustratif, on peut penser que les services téléphoniques d'affichage du numéro d'appel ont eu un effet désolant chez les harceleurs téléphoniques.

Quant aux différents tribunaux et services correctionnels de notre pays, il ne faudrait pas que des baisses de la criminalité servent de prétexte à une diminution de l'intensité de la punition infligée aux délinquants (par exemple, par une plus grande utilisation de la libération conditionnelle). Actuellement, les détenus ne purgent qu'une portion du temps que leur a imposé un juge et ceux-ci, de l'avis du public, s'en tirent bien ${ }^{10}$. D'autre part, il y a un besoin de spécialisation des soins à apporter aux criminels durant leur détention: certains ont des besoins de traitement psychologique, d'autres demandent une aide pour combattre leur problèmes de toxicomanies. De manière plus générale, nous croyons qu'en matière de vol et surtout de violence, il n'y a pas lieu d'être particulièrement tolérant avec les criminel adultes qui en sont à leur deuxième ou troisième condamnation. Un message clair doit être envoyé aux délinquants potentiels.

Si l'efficacité éventuelle de tels aménagements est mise en doute, on a qu'à regarder, à titre d'exemple, la stratégie de lutte contre l'alcool au volant menée un peu partout durant les années quatre-vingt. La lutte prenait place sur trois front : a) une campagne de publicité pour

10 Tremblay Pierre, 1994, La justice sondée: tribunaux criminels, décisions sentencielles et opinion publique. In: Denis Szabo et Marc LeBlanc, Traité de criminologie empirique. Montréal: Les Presses de l'Université de Montréal. 
dénoncer les dommages causés par cette conduite (et ainsi changer les valeurs), b) un resserrement des contrôles policiers et un durcissement des peines et c) une pression administrative effectuée par les bureaux des licences (points de démérite, suspension de permis...). On peut maintenant voir les fruits de cette stratégie en trois points: le nombre de décès sur les routes a chuté au cours de la dernière décennie. Il est possible que ce genre d'action concertée pourrait être mise de l'avant rapidement pour des conduites comme la violence familiale ou l'abus sexuel.

Fin du texte 